\title{
Biquaternionic Form of Laws of Electro-Gravimagnetic Charges and Currents Interactions
}

\author{
L. A. Alexeyeva \\ Institute of Mathematics and Mathematical Modeling, Almaty, Kazakhstan \\ Email: alexeeva@math.kz
}

Received 8 June 2016; accepted 24 July 2016; published 27 July 2016

Copyright (C) 2016 by author and Scientific Research Publishing Inc.

This work is licensed under the Creative Commons Attribution International License (CC BY).

http://creativecommons.org/licenses/by/4.0/

(c) (i) Open Access

\begin{abstract}
One the base of differential algebra of biquaternions, the one model of electro-gravimagnetic interactions of electric and gravimagnetic charges and currents has been constructed. For this, three Newton laws analogues are used. The closed system of biquaternionic wave equations is constructed for determination of the charges-currents and electro-gravimagnetic fields and united field of interactions. The equation of charge-current transformation is like the generalization of biquaternionic presentation of Dirac equation. The properties of its solutions are described, depending on properties of external EGM field. The biquaternions of energy-pulse of EGM-field and charges-currents are considered. The energy-pulse of EGM-interactions is calculated.
\end{abstract}

\section{Keywords}

Electro-Gravimagnetic Field, Electric Charge, Gravimagnetic Charge, Current, Energy, Biquaternion, Bigradient, Dirac Equation, Newton Laws

\section{Introduction}

In the paper [1], we described the one biquaternionic model of electro-gravimagnetic (EGM) field, charges and currents. In this model, gravitational field (which is potential) is united with magnetic field (which is torsional) which gives possibility to enter gravimagnetic tension, gravimagnetic charge and gravimagnetic current. There we have shown that in algebra of biquaternions, the charges and currents of EGM-field are physical appearance of bigradient of EGM-intensity. Differential operator bigradient is the generalization of gradient operator on the space of biquaternions which characterizes a direction of more extensive change of biquaternionic functions. If bigradient of EGM-intensity is equal to zero then charges and currents are absent. Also there we constructed the 
law of inertia for a free system of mass, charges and currents, which described their motion under action only with internal electric and gravimagnetic tensions. This law is the fields analogue of the first Newton lawinertia law for a solid (considered as material point).

Here we consider the motion of electro-gravimagnetic charges and currents under action of external EGMfields which are created by other charges and currents. The laws of their interaction are the fields analogue of the second and third Newton laws. They have been constructed in the form of biquaternionic wave equations which generalize biquaternionic form of Dirac equations. Some solutions of them are discussed. The energypulse of EGM-interactions is calculated.

We used also here the differential algebra of biquaternions in Hamiltonian form which was shortly described in [1] (see [2] for more detail). This form is very convenient for description of physical fields. There are voluminous literature about application of algebras of quaternions and biquaternions in fields theory, in the theory of electromagnetic fields, and quantum mechanics [3]-[17]. Differential algebra of biquaternions gives possibility to simplify mathematical record of systems of Maxwell and Dirac equations to construct their solutions and to study their properties.

The novelty of this work is the construction of laws of electro-gravymagnetic interactions on the base of biquaternions algebra. The properties of this algebra and operation of quaternionic multiplication in fields theory have visual physical interpretation and detect new objective laws which are impossible to determine without this algebra. It's natural for matter as you'll see here.

\section{Biquaternions of Electro-Gravimagnetic Field, Charges and Currents}

Their are the next complex characteristics of EGM-field [1]:

- complex vector of EGM-intensity

$$
A(\tau, x)=A^{E}+i A^{H}=\sqrt{\varepsilon} E+i \sqrt{\mu} H
$$

- complex charges field:

$$
\rho(\tau, x)=\rho^{E} / \sqrt{\varepsilon}-i \rho^{H} / \sqrt{\mu} \text {; }
$$

- complex currents field:

$$
J(\tau, x)=\sqrt{\mu} j^{E}-i \sqrt{\varepsilon} j^{H} ;
$$

- complex scalar field of attraction-resistance

$$
\alpha(\tau, x)=\frac{i \alpha_{1}}{\sqrt{\varepsilon}}+\frac{\alpha_{2}}{\sqrt{\mu}} .
$$

Here real vectors $E$ and $H$ are the tensions of electric and gravimagnetic fields; real scalars $\rho^{E}, \rho^{H}$ are the densities of electric and gravimagnetic charges; real vectors $j^{E}, j^{H}$ are the densities of electric and gravimagnetic currents; values $\varepsilon, \mu$ are the constants of electric conductivity and magnetic permeability of the EGM-medium.

In biquaternions algebra on Minkowski space $\mathbb{M}=\{(\tau, x)\}$ the EGM-field, charges and currents may be presented by use the next biquaternions:

EGM-intensity

$$
\mathrm{A}(\tau, x)=i \alpha(\tau, x)+A(\tau, x)
$$

charge-current

$$
\Theta(\tau, x)=-i \rho(\tau, x)-J(\tau, x) .
$$

In the paper [1] [2] was shown that connection between EGM-intensity and charge-current has the bigradiental form:

POSTULATE 1

$$
\nabla^{+} \mathbf{A}=\left(\partial_{\tau}+i \nabla\right) \circ \mathbf{A}=\Theta(\tau, \mathbf{x}) .
$$

Here and further the bigradients $\nabla^{+}$and $\nabla^{-}$are the next biquaternionic differential operators: 


$$
\begin{aligned}
\nabla^{ \pm} \mathbf{F} & \triangleq\left(\partial_{\tau} \pm i \nabla\right) \circ(f+F)=\left(\partial_{\tau} f \mp i(\nabla, F)\right) \pm \partial_{\tau} F \pm i \nabla f \pm i[\nabla, F] \\
& =\left(\partial_{\tau} f \mp i \operatorname{div} F\right) \pm \partial_{\tau} F \pm \text { igrad } f \pm i r o t F .
\end{aligned}
$$

Further we name them mutual bigradients. They define a "directions" of more intensive changing of biquaternionic field.

\section{The Power and Density of Acting Forces}

Let's consider two systems of charges and currents $\Theta(\tau, x), \Theta^{\prime}(\tau, x)$. Every of them generate own EGM-field: $\mathbf{A}(\tau, x)=i a+A$ and $\mathbf{A}^{\prime}(\tau, x)=i a^{\prime}+A^{\prime}$, which corresponds to (1). At first let consider the case when $a^{\prime}=0$. We name a power-force density the next biquaternion

$$
\mathbf{F}=p-i F=\mathbf{A}^{\prime} \circ \Theta=-A^{\prime} \circ(i \rho+J)=\left(A^{\prime}, J\right)-i \rho A^{\prime}-\left[A^{\prime}, J\right]
$$

which is acting from side of $A^{\prime}$-field on the charge and current of $A$-field. Really, according to definitions, the scalar part is determined as power density of acting forces:

$$
p=\left(A^{\prime}, J\right)=c^{-1}\left(\left(E^{\prime}, j^{E}\right)+\left(H^{\prime}, j^{H}\right)\right)+i\left(\left(B^{\prime}, j^{E}\right)-\left(D^{\prime}, j^{H}\right)\right) .
$$

Here $B=\mu H$ is analogue of a magnetic induction (in torsional part complies with it), $D=\varepsilon E$ is a vector of an electric offset.

Selecting the real and imaginary part from the formulae (2) we get the expressions for a density of acting forces $\left(F=F^{H}+i F^{E}\right)$ :

$$
\begin{aligned}
& F^{H}=\rho^{E} E^{\prime}+\rho^{H} H^{\prime}+B^{\prime} \times j^{E}-D^{\prime} \times j^{H} \\
& F^{E}=c\left(\rho^{E} B^{\prime}-\rho^{H} D^{\prime}\right)-c^{-1}\left(E^{\prime} \times j^{E}+H^{\prime} \times j^{H}\right) .
\end{aligned}
$$

Potentional part of $H^{\prime}$ describes the tension of gravitational field. Torsional part of this vector describes magnetic field. The scalar part of $\Theta, \Theta^{\prime}$ contains the densities of electric charge and gravitational mass. Its vector part contains the densities of electric and mass currents. Coming from these suggestions, in formula (4) we see the known forces, appropriately:

- Coulomb's force $\rho^{E} E^{\prime}$;

- gravimagnetic force $\rho^{H} H^{\prime}$ (it complies with gravitational force in a potential part $H^{\prime}$ );

- Lorentz force $B^{\prime} \times j^{E}$ (more exactly, it complies with it in torsional part $B^{\prime}$ );

- gravielectric force $j^{H} \times D^{\prime}$.

In real part of the power $p$ (3) we see the powers of Coulomb's force, gravitational and magnetic forces. The power of gravielectric force in real part of (3) does not enter as it does not work on the mass displacement, because it is perpendicular to its velocity. It's interesting that Lorentz force also does not enter in real part of (3). It proves that this force is perpendicular to mass velocity, though directly from Maxwell equations this does not follow.

Naturally, by analogy, we assume that formula (5) describes forces, causing a change of electric current. Consequently their power stands in imaginary part $p$ (3).

With entering the scalar field $a^{\prime}$, type of scalar and vector parts of power-force biquaternion (2) is changed, as follows

$$
\mathbf{F}=\mathbf{A}^{\prime} \circ \Theta=\left(\left(A^{\prime}, J\right)+a^{\prime} \rho\right)-i\left(a^{\prime} J+\rho A^{\prime}\right)-\left[A^{\prime}, J\right]
$$

You see here the additional summands which appear in presentation of the powers $\left(a^{\prime} \rho\right)$ and force $\left(-i a^{\prime} J\right)$.

Vector $a^{\prime} J$ describes absorbtion-resistance force which acts on $\Theta$ from $A^{\prime}$-field.

\section{CC-Transformations Equation: Second Newton Law}

The charge-current field $\Theta$ is changed under influence of the $\mathbf{A}^{\prime}$-field. As it's well known, the direction of the most intensive change the scalar field describes its gradient. By analogy we expect that change of chargecurrent biquaternion is more intensive toward its bigradient. Naturally to expect that this change must be toward external power-force. 
POSTULATE 2. The law of a change of a charge-current field under the action of external EGM-field is

$$
\kappa \nabla^{-} \boldsymbol{\Theta}=\mathbf{F} \triangleq \mathbf{A}^{\prime} \circ \Theta .
$$

Entering the constant of interaction $\kappa$ is connected with dimensionality. We name Equation (7) as $C C$ transformations equation.

If one EGM-field much stronger then second one:

$$
\left\|\mathbf{A}^{\prime}\right\| \gg\|\mathbf{A}\|
$$

it's possible to neglect the second field change under influence of charge and current on $\mathbf{A}^{\prime}$-field. In this case from Equation (7) we can find the CC-field $\Theta(\tau, \mathbf{x})$, its changing under action of external $\mathbf{A}^{\prime}$-field.

Revealing scalar and vector part from (7), we get the system of two differential equations:

$$
\begin{gathered}
i \kappa\left(\partial_{\tau} \rho+\operatorname{div} J\right)=\left(A^{\prime}, J\right)+a^{\prime} \rho=p, \\
i \kappa\left(\partial_{\tau} J-i r o t J+\nabla \rho\right)=\left(a^{\prime} J+\rho A^{\prime}\right)-i\left[A^{\prime}, J\right]=F .
\end{gathered}
$$

By use (3), (4) we obtain from Equation (9) the two vectorial differential equations:

$$
\begin{aligned}
& \kappa\left(\sqrt{\varepsilon} \partial_{\tau} j^{H}+\sqrt{\mu} \operatorname{rot} j^{E}+\mu^{-0.5} \operatorname{grad} \rho^{H}\right)=\rho^{E} E^{\prime}+\rho^{H} H^{\prime}+B^{\prime} \times j^{E}-D^{\prime} \times j^{H}+\operatorname{Re}\left(a^{\prime} J\right), \\
& \kappa\left(\sqrt{\mu} \partial_{\tau} j^{E}-\sqrt{\varepsilon} \operatorname{rot} j^{H}+\varepsilon^{-0.5} \operatorname{grad} \rho^{E}\right)=c\left(\rho^{E} B^{\prime}-\rho^{H} D^{\prime}\right)-c^{-1}\left(E^{\prime} \times j^{E}+H^{\prime} \times j^{H}\right)+\operatorname{Im}\left(a^{\prime} J\right) .
\end{aligned}
$$

The Equation (10) is the second Newton law analogue for CC-field. Here the value $\kappa \sqrt{\varepsilon} j^{H}$ is analogue of mass momentum. In right part you see all known forces but also two new forces: gravielectric force and absorbtion-resistance force. Last of them is proportional to currents. Their direction depends on signs of real and imaginary part $a^{\prime}(\tau, x)$ which can be as positive and negative. By analogy to mechanics of media we call it such name.

Equation (10) describes the motion of gravimagnetic charges and currents under action of the external EGMfield. Consequently Equation (11) defines the motion of electric charges and currents.

The scalar Equation (8) is the law of conservation of electric and gravimagnetic charges. As you see the external EGM-field can essentially change CC-field.

\section{Third Newton Law: The Laws of Charge-Currents Interactions}

On the virtue of the third Newton law about acting and counteracting forces, we suppose that must be executed for electro-gravimagnetic forces the equality:

POSTULATE 3

$$
\mathbf{F}^{\prime}=-\mathbf{F} .
$$

From here we get

fields analogue of third Newton law:

$$
\mathbf{A}^{\prime} \circ \Theta=-\mathbf{A} \circ \Theta^{\prime} .
$$

By use it we construct

the law of the charge-current interaction:

$$
\begin{aligned}
& \kappa \nabla^{-} \Theta=\mathbf{A}^{\prime} \circ \Theta, \kappa \nabla^{-} \Theta^{\prime}=\mathbf{A} \circ \Theta^{\prime}, \\
& \mathbf{A}^{\prime} \circ \Theta=-\mathbf{A} \circ \Theta^{\prime}, \\
& \nabla^{+} \mathbf{A}=\Theta, \nabla^{+} \mathbf{A}^{\prime}=\Theta^{\prime} .
\end{aligned}
$$

Here Equation (13) correspond to the second Newton law which is written for charge-current each of interacting field. Equation (14) is the third Newton law. Together with Maxwell equations for these fields (15) they give closed system of the nonlinear differential equations for determination $\mathbf{A}, \mathbf{A}^{\prime}, \Theta, \Theta^{\prime}$.

It is interesting that in scalar part of Equation (12) it requires the equality of the powers corresponding to forces, acting on charges and currents of the other field, i.e. it is befitted known in mechanics the identity to reciprocity of Betty, which is usually written for the forces works. 


\section{First Newton Law: Free EGM-Field}

Let's consider A-field, which is generated $\Theta$, in absence of other charges-currents. We name it $a$ free field. In this case, $\mathbf{F}=0$. From (13) we get inertia law, which is analogue of the first Newton law for charge-current:

$$
\nabla^{-} \Theta=\mathbf{0},
$$

which is equivalent to equations:

$$
\partial_{\tau} \rho+\operatorname{div} J=0, \partial_{\tau} J-i \operatorname{rot} J+\nabla \rho=0 .
$$

For initial designations we have following formulas:

$$
\begin{gathered}
\partial_{t} \rho^{E}+\operatorname{div} j^{E}=0, \quad \partial_{t} \rho^{H}+\operatorname{div} j^{H}=0 ; \\
\partial_{\tau} j^{E}=\sqrt{\varepsilon / \mu} \operatorname{rot} j^{H}-\operatorname{cgrad} \rho^{E}, \quad \partial_{\tau} j^{H}=-\sqrt{\mu / \varepsilon} \operatorname{rot} j^{E}-\operatorname{cgrad} \rho^{H} .
\end{gathered}
$$

Naturally that the well known law of charges conservation in the form (17) must be executed in absence of external EGM-field.

This law (16) was considered in [1] and solutions of this equations were defined in the next form:

$$
\Theta=\nabla^{-} \psi^{0}+i \sum_{j=1}^{3} \nabla^{-}\left(\psi^{j} e_{j}\right) .
$$

Scalar potentials $\psi^{j}$ are arbitrary solutions of classic wave equation:

$$
\frac{\partial^{2} \psi^{j}}{\partial \tau^{2}}-\Delta \psi^{j}=0, \quad j=0,1,2,3,4
$$

where $\Delta$ is Laplace operator. They may be presented so

$$
\psi^{j}=\int_{R^{3}} \varphi^{j}(\xi) \exp (-i(\xi, x)-i\|\xi\| t) \mathrm{d} \xi_{1} \mathrm{~d} \xi_{2} \mathrm{~d} \xi_{3}, \quad \forall \varphi^{j}(\xi) \in L_{1}\left(R^{3}\right) .
$$

We give here also for (16) the solution of Cauchy problem

$$
\Theta(\tau, x)=-\frac{H(\tau)}{4 \pi} \nabla^{+}\left\{\tau^{-1} \int_{\|y-x\|=\tau} \Theta_{0}(y) \mathrm{d} S(y)\right\},
$$

were there are the initial conditions:

$$
\Theta(0, x)=\Theta_{0}(x), \mathbf{A}(0, x)=\mathbf{A}_{0}(x) .
$$

From postulate 1 to follow that A-field is defined in the form:

$$
4 \pi A(\tau, x)=-\nabla^{-}\left\{\int_{r \leq \tau} \frac{\Theta(\tau-r, y)}{r} \mathrm{~d} V(y)+\tau^{-1} \int_{r=\tau} \mathbf{A}_{0}(y) \mathrm{d} S(y)\right\}-\tau^{-1} \int_{r=\tau} \boldsymbol{\Theta}(0, y) \mathrm{d} S(y),
$$

where $r=\|y-x\|, \mathrm{d} V(y)=\mathrm{d} y_{1} \mathrm{~d} y_{2} \mathrm{~d} y_{3}, \mathrm{~d} S(y)$ is a differential of spheres area. About determination of these solutions see the theory of biwave equation in [2].

This formula is a generalization of the famous Kirchhoff formula for solution of Cauchy problem for wave equation [18].

\section{The Solutions of CC-Transformation Equation by Action of Invariable External EGM-Field}

Let consider Equation (7) when external EGM-field $\mathbf{A}^{\prime}$ is constant, don't depend on $\tau$ and $x$. We write it in the form

$$
\nabla^{-} \Theta-\kappa^{-1} \mathbf{A}^{\prime} \circ \Theta=0 .
$$

This equation is biquaternionic generalization of Dirac equations. Its presentation by use differential equations 
coincides with Dirac equations when $\mathbf{A}^{\prime}=i m, m$ is real constant. About construction full system of solutions of homogeneous and inhomogeneous Dirac equation see [4] [5].

There are simple connection between solutions of Equation (7) and Equation (22):

$$
\Theta=\Theta_{0} \exp \left(\kappa^{-1}\left(a^{\prime} \tau+i\left(A^{\prime}, x\right)\right)\right)
$$

where $\Theta_{0}$ is the solution of (7):

$$
\nabla^{-} \Theta_{0}=0 .
$$

From here to follow that imaginary part of scalar field resistance-absorbtion $a^{\prime}$ creates harmonic vibration with frequency $a_{1} / \sqrt{\varepsilon}$, but real part ' increases or decreases $\left\|\Theta_{0}\right\|$ over time depend on a sign $a_{2}$. Real part of vector $A^{\prime}$ (electric field) creates for $\Theta_{0}$ sinusoidal deviation in $R^{3}$ along direction of $E$. But its imaginary part (gravymagnetic field) gives exponential growth or diminution $\|\Theta\|$ along direction the vector $H$.

\section{Energy-Impulse Biquaternions: First Thermodynamics Law}

We introduce the biquaternion of energy-impulse of EGM-field ( EGM-energy-impulse):

$$
W+i P=0.5 \mathbf{A}^{*} \circ \mathbf{A},
$$

where $\mathbf{A}^{*}$ is conjugated $\mathbf{A}$ :

$$
\mathbf{A}=a+A, \quad \mathbf{A}^{*}=\bar{a}-\bar{A}=-i \bar{\alpha}-\bar{A},
$$

$\bar{a}, \bar{A}$ are complex conjugated to $a, A$. Here positive scalar function $W$ is energy density of EGM field:

$$
W=0.5\left(\|\alpha\|^{2}+\|A\|^{2}\right)=0.5\left(\|\alpha\|^{2}+\|E\|^{2}+\|H\|^{2}\right),
$$

$P$ is real vector-function:

$$
P=0.5(a \bar{A}-\bar{a} A+[A, \bar{A}])=\alpha_{2} E / \sqrt{\mu}+\alpha_{1} H / \sqrt{\varepsilon}+[E, H] .
$$

By $\alpha=0$, as you see, $W, P$ are like to an energy and Pointing vector of EM-field and satisfid to equation:

$$
\partial_{\tau} W+\operatorname{div} P=-\operatorname{Re}(J, \bar{A})=c^{-1}\left(j^{H} H-j^{E} E\right) .
$$

By analogue we construct the biquaternion of energy-impulse for charge-current field ( CC-energy-impulse):

$$
0.5 \Theta^{*} \circ \Theta=\left(\frac{\left\|\rho_{E}\right\|^{2}}{\varepsilon}+\frac{\left\|\rho_{H}\right\|^{2}}{\mu}+Q\right)+i\left(P_{J}-\sqrt{\frac{\mu}{\varepsilon}} \rho^{E} j^{E}-\sqrt{\frac{\varepsilon}{\mu}} \rho^{H} j^{H}\right) .
$$

It contains currents energy :

$$
Q=0.5\|J\|^{2}=0.5\left(\mu\left\|j^{E}\right\|^{2}+\varepsilon\left\|j^{H}\right\|^{2}\right),
$$

where first summand includes Joule heat $\left\|j^{E}\right\|^{2}$; second one includes kinetic energy density of mass current $\left\|j^{H}\right\|^{2}$, also it contains the energy of torsional part of currents (magnetic current). Here vector $P_{J}$ is analogue of Pointing vector, but for the current:

$$
P_{J}=0.5 i J \times \bar{J}=c^{-1}\left[j^{E}, j^{H}\right] .
$$

Only if gravimagnetic and electrical currents are parallel or one from them is equal zero, then $P_{J}=0$. If to take scalar product Equation (9) with $i \bar{J}$, we get

$$
\kappa\left(\partial_{\tau} Q-\operatorname{div} P_{J}+\operatorname{Re}(\nabla \rho, \bar{J})\right)=\operatorname{Im}(F, \bar{J})=c^{-1}\left(\left(F^{H}, j^{H}\right)+\left(F^{E}, j^{E}\right)\right) .
$$

It is easy to see that this law is like to the first thermodynamics law. Here the sum of second and third summands in left part is designated $-U$. The function

$$
U=\operatorname{div} P_{J}-\sqrt{\mu / \varepsilon}\left(\nabla \rho^{E}, j^{E}\right)-\sqrt{\varepsilon / \mu}\left(\nabla \rho^{H}, j^{H}\right)
$$


characterizes the own velocity of the change of current energy of $\Theta$-field. Right part (30), which depends on power of acting external forces, can to increase or decrease this velocity.

If there are not acting external forces,

$$
\partial_{\tau} Q=U .
$$

It's the first thermodynamics law for a free CC-field.

\section{The United Field of Interaction: Energy-Pulse of Interactions}

If there are some $(N)$ interacting CC-fields then we have for every of them

$$
\begin{aligned}
& \kappa \nabla^{-} \Theta^{k}=\sum_{m \neq k} \mathbf{A}^{m} \circ \Theta^{k}, \nabla^{+} \mathbf{A}^{k}=\Theta^{k}, \quad k=1, \cdots, N \\
& \nabla^{+} \mathbf{A}^{m} \circ \mathbf{A}^{k}=-\nabla^{+} \mathbf{A}^{k} \circ \mathbf{A}^{m}, \quad k \neq m .
\end{aligned}
$$

United CC-field, as easy to see after summing the first equation over $k$, is free. It satisfies to the inertia law

$$
\nabla^{-} \Theta=\nabla^{-} \sum_{m=1}^{M} \Theta^{m}=\mathbf{0}
$$

because all forces are internal, also as in Newton mechanics of interacting solids.

Let consider the laws of energy transformation at interaction of different charges-currents. Energy-pulse for united charge-current field has the form:

$$
\boldsymbol{\Xi}_{\Theta}=0.5 \Theta \circ \Theta^{*}=0.5 \sum_{k=1}^{N} \Theta^{k} \circ \sum_{l=1}^{N} \Theta^{* l}=0.5\left(\sum_{k=1}^{N} \Theta^{k} \circ \Theta^{* k}+\sum_{k \neq l} \Theta^{k} \circ \Theta^{* l}\right)=\sum_{k=1}^{N} W_{\Theta}^{(k)}+i \sum_{k=1}^{N} P_{\Theta}^{(k)}+\delta \boldsymbol{\Xi}_{\Theta} .
$$

Here the first summand is an amount of energy-pulse of interacting charge-current.

We introduce biquaternion of energy-pulse interaction. Its real part describes energy-pulse interaction for the same name charge and current, but imagine part for different name ones:

$$
\begin{gathered}
\delta \boldsymbol{\Xi}_{\Theta}=\delta W_{\Theta}+i \delta P_{\Theta}=\sum_{k \neq l} \Xi_{\Theta}^{k l}, \quad \Xi_{\Theta}^{k l}=0.5\left(\Theta^{k} \circ \Theta^{* l}+\Theta^{l} \circ \Theta^{* k}\right) \\
\Xi_{\Theta}^{k l}=\operatorname{Re}\left(\rho^{k} \rho^{* l}+\left(J^{k}, J^{* l}\right)\right)-i\left\{\operatorname{Re}\left(\rho^{k} J^{* l}+\rho^{* l} J^{k}\right)+\operatorname{Im}\left[J^{k}, J^{* l}\right]\right\},
\end{gathered}
$$

or in initial designations:

$$
\begin{aligned}
\Xi_{\Theta}^{k l}= & \frac{\rho^{E(k)} \rho^{E(l)}}{\sqrt{\varepsilon_{k} \varepsilon_{l}}}+\frac{\rho^{(k) H} \rho^{H(l)}}{\sqrt{\mu_{k} \mu_{l}}}+\sqrt{\mu_{k} \mu_{l}}\left(j^{(k) E}, j^{(l) E}\right)+\sqrt{\varepsilon_{k} \varepsilon_{l}}\left(j^{(k) H}, j^{(l) H}\right) \\
& -i\left\{\sqrt{\frac{\mu_{l}}{\varepsilon_{k}}} \rho^{(k) E} j^{(l) E}+\sqrt{\frac{\varepsilon_{l}}{\mu_{k}}} \rho^{(k) H} j^{(l) H}+\sqrt{\frac{\mu_{k}}{\varepsilon_{l}}} \rho^{(l) E} j^{(k) E}+\sqrt{\frac{\varepsilon_{k}}{\mu_{l}}} \rho^{(l) H} j^{(k) H}\right. \\
& \left.-\sqrt{\varepsilon_{k} \mu_{l}}\left[j^{(l) E}, j^{(k) H}\right]+\sqrt{\varepsilon_{l} \mu_{k}}\left[j^{(k) E}, j^{(l) H}\right]\right\} .
\end{aligned}
$$

As result we get the conditions of energy transformation by charges-currents interaction:

energy separation if $\delta W_{\Theta}>0$;

energy absorption if $\delta W_{\Theta}<0$;

energy conservation if $\delta \boldsymbol{\Xi}_{\Theta}=0$.

Vector $\delta P_{\Theta}$ shows the main direction and intensities of these energy processes.

\section{Conclusions}

We construct here biquaternionic forms of laws of electric and gravimagnetic charges and currents interaction by analogy to Newton laws, which gives us closed hyperbolic system of differential equations for their definition and determination of corresponding EGM-fields. For the free system of charges and currents, Equations (22) and (24) define the behavior of CC-field and EGM-field over time if their initial states are known. After calculating 
the bigradients from here, the all fields, charges and currents can be defined according to their definitions. It's the very suitable short form which contains the algorithm for their calculation.

At building of charge-current transformations equation, we get as known gravitational, electric and magnetic forces, so we found the new forces which are needed in experimental motivation. Considered properties (26) of solutions of CC-transformation equation by existence external EGM-field give possibility to test this model on practice.

Note also that the essential at building and studying this model of EGM-field and CC-field is the differential algebra of biquaternions [2], without which such construction of differential equations, describing interaction of charges and currents in the forms which give the fields analogies of Newton laws and are very convenient for calculations, will be practically impossible.

\section{References}

[1] Alexeyeva, L.A. (2016) Journal of Modern Physics, 7, 435-444. http://www.scirp.org/journal/jmp http://dx.doi.org/10.4236/jmp.2016.75045

[2] Alexeyeva, L.A. (2012) Int. J. Clifford Analysis, Clifford Algebras and Their Applications, 7, 19-39.

[3] Rastall, R. (1964) Review of Modern Physics, 36, 820-832. http://dx.doi.org/10.1103/RevModPhys.36.820

[4] Edmonds Jr., J.D. (1978) American Journal of Physics, 46, 430. http://dx.doi.org/10.1119/1.11316

[5] Spilker, G.L. (1983) Report of USSR Academy of Sciences, 272, 1359-1363.

[6] Rotelli, P. (1989) Modern Physics Letters A, 4, 933-940. http://dx.doi.org/10.1142/S0217732389001106

[7] Davies, A.J. (1990) Physical Review D, 41, 2628-2630. http://dx.doi.org/10.1103/PhysRevD.41.2628

[8] Finkelstein, D., Jauch, J.M., Schiminovich, S. and Speiser, D. (1992) Journal of Mathematical Physics, 3, $207-220$. http://dx.doi.org/10.1063/1.1703794

[9] Adler, S.L. (1995) Quaternionic Quantum Mechanics and Quantum Fields. Oxford University Press, New York.

[10] Kravchenko, V.V. (1995) Doklady Mathematics, 51, 287-289.

[11] Kassandrov, V.V. (1995) Gravitation and Cosmology, 1, 216-222.

[12] De Leo, S. and Rodrigues Jr., S. (1998) International Journal of Theoretical Physics, 37, 1707-1720. http://dx.doi.org/10.1023/A:1026692508708

[13] Kravchenko, V.V. (2003) Quaternionic Equation for Electromagnetic Fields in Inhomogeneous Media. In: Begehr, H., Gilbert, R. and Wah Wong, M., Eds., Progress in Analysis, Vol. 1, World Scientific, 361-366.

[14] Efremov, A.P. (2004) Hypercomples Numbers in Geometry and Physics, 1, 111-127.

[15] Acevedo, M., Lopez-Bonilla, J. and Sanchez-Meraz, M. (2005) Apeiron, 12, 371.

[16] Alexeyeva, L.A. (2013) Differential Algebra of Biquaternions. Dirac Equation and Its Generalized Solutions. Progress in Analysis. Proceedings of the 8th Congress of the ISAAC, Moscow, 22-27 August 2013, 153-161.

[17] Alexeyeva, L.A. (2013) Mathematical Journal, 13, 17-35. (In Russian) http://www.math.kz/images/journal/2013-1/Alexeyeva.pdf

[18] Vladimirov, V.S. (1976) Generalized Functions in Mathematical Physics. Nauka Publisher, Moscow. 


\section{Submit or recommend next manuscript to SCIRP and we will provide best service for you:}

Accepting pre-submission inquiries through Email, Facebook, LinkedIn, Twitter, etc.

A wide selection of journals (inclusive of 9 subjects, more than 200 journals)

Providing 24-hour high-quality service

User-friendly online submission system

Fair and swift peer-review system

Efficient typesetting and proofreading procedure

Display of the result of downloads and visits, as well as the number of cited articles

Maximum dissemination of your research work

Submit your manuscript at: http://papersubmission.scirp.org/ 